\title{
Allergo Journal im zehnten Jahrgang
}

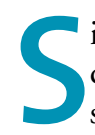
ie werden sich wundern, warum dieses „Zur Sache" nur eine Unterschrift trägt. Der Grund ist einfach: Ich will mich mit diesem Heft von Ihnen als Chef-Herausgeber verabschieden. Die Entscheidung ist mir nicht leicht gefallen, aber neue Verpflichtungen machen diesen Schritt notwendig.

Die Gründung von ALLERGO JOURNAL im Jahr 1992 war ein Wagnis, dem sich der Vorstand der DGAI damals einstimmig gestellt hat. Mit dieser Zeitschrift sollten zwei Ziele erreicht werden: zum Ersten ein qualitativ hochwertiger Originalienteil, bei dem auch internationale Autoren auf Englisch zur Sprache kommen können, zum Zweiten ein aktuelles Forum. Trotz Erfüllung aller Kriterien im Originalienteil sind wir aber leider noch nicht in "Medline“. Wer die einzelnen Bände seit 1992 durchblättert, wird eine ganze Reihe origineller Beiträge finden mit Meilensteincharakter für die deutsche Allergie-Forschung. Ich danke allen Autoren, die den Verlust an „Impact-Punkten“ dafür in Kauf genommen haben.

Das zweite Ziel, ein Forum für die aktuellen Probleme der praktischen Allergologie im deutschsprachigen Raum zu schaffen, ist auf enorme Resonanz Zeit für einen Wechsel... gestoßen. Kongressberichte, Fortbildung, Standespolitik, praktische Tipps aus der Industrie, Positionspapiere, Leitlinien, aber auch Leserbriefe mit durchaus heftiger Kontroverse (Stichwort „Raubmilbenkot"), machen das ALLERGO JOURNAL lebendig.

ALLERGO JOURNAL wurde vom Vorstand der DGAI unter der Präsidentschaft von Gert Kunkel ins Leben gerufen. Ich danke ihm, dem gesamten damaligen Vorstand und unserem Ehrenherausgeber Erich Fuchs für die Unterstützung. Etwas später hat sich auch der Ärzteverband Deutscher Allergologen (ÄDA) angeschlossen. Die Zeitschrift ist

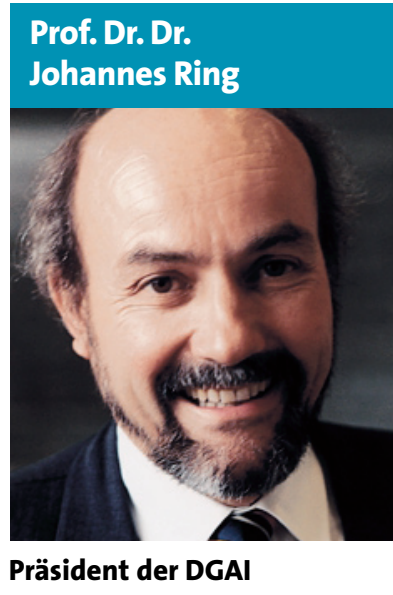

wichtig für unsere Gesellschaften! Sie spiegelt das Leben und den Stand von Forschung und Praxis in der Allergologie in Deutschland wieder. Mit einer gedruckten Auflage von über 7.000 hat sich ALLERGO JOURNAL fest im deutschen Raum etabliert. Eine noch größere Zahl von Abonnenten würde die Unabhängigkeit gegenüber der Industrie stärken! Deshalb mein Appell an interessierte Leser: Werden Sie Mitglied einer der beiden Gesellschaften; ALLERGO JOURNAL ist im Mitgliedsbeitrag eingeschlossen. Für Studenten und AiPs gibt es bei der DGAI drei Jahre Mitgliedschaft und ALLERGO JOURNAL zum halben Preis.

Durch unser Sprachrohr haben wir einiges zur Verbesserung der Situation Allergie-kranker Menschen in Deutschland erreicht. Entscheidende Positionspapiere wie das zur „Allergie-Prävention “, zum "Stand der Allergie-Forschung in Deutschland“ oder zur „Latex-Allergie " hatten großen politischen Impact und haben u.a. zur Ausschreibung großer Schwerpunktprogramme durch die Bundesregierung beigetragen.

ALLERGO JOURNAL hat ein unverwechselbares Gesicht, nicht zuletzt durch die Titelseite mit den über neun Jahre hinweg wissenschaftlich und ästhetisch erstklassigen elektronenoptischen Abbildungen, fast ausschließlich von Heidrun Behrendt; dafür an dieser Stelle vielen Dank! Dank auch an das Herausgeber-Kollegium für wertvolle Ideen und Mitarbeit!

Als Pate und Geburtshelfer gegenwärtig bei Zeugung, Schwangerschaft, Geburt und Taufe war Jochen Aumiller, der langjährige Chefredakteur der Münchner Medizinischen Wochenschrift und unserer Zeitschrift, bei dem ich mich ebenfalls herzlich bedanken möchte.

Ganz besonderer Dank geht jedoch in dieser Stunde an den Mit-Herausgeber Gerhard Schultze-Werninghaus. Ich habe unsere Zusammenarbeit auf vielen Gebieten als nahezu ideal und immer erfreulich empfunden. Diese problemlose und auf ähn- ... und für einen licher Wellenlänge sach- Rückblick orientierte Kooperation zwischen Vertretern von Haut und Atemwegen stellte eine Garantie für ein funktionierendes Journal auf dem interdisziplinären Gebiet der Allergologie dar.

Ich bin deshalb außerordentlich glücklich, dass es gelungen ist, mit Bernhard Przybilla einen Nachfolger zu finden, der in der Kooperation mit Gerhard Schultze-Werninghaus die Kontinuität von ALLERGO JOURNAL in wesentlichen Punkten gewährleisten wird. Ich selbst werde dem HerausgeberKollegium als Vertreter der DGAI erhalten bleiben.

Allergo Journal ist aus dem Kleinkindes- ins Schulalter gekommen. Allerdings steht ihm die Pubertät und das Erwachsenwerden immer noch bevor. Es wird aufregend sein, die weitere Entwicklung zu beobachten und zu unterstützen. Ich wünsche den Kollegen Schultze-Werninghaus und Przybilla viel Erfolg!

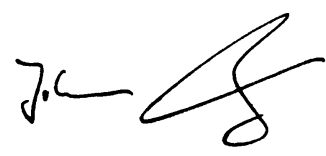

Prof. Dr. Dr. J. Ring 